\title{
DEMOCRACIA Y PARTICIPACIÓN CIUDADANA EN LOS MUNICIPIOS: ¿UN MERCADO POLÍTICO DE TRASTOS ${ }^{1}$ ?
}

\author{
Fernando Aguiar \\ IESA de Andalucía/CSIC \\ E-mail: faguiar@iesaa.csic.es \\ Clemente J. Navarro \\ Universidad Pablo de Olavide \\ E-mail: cnavyan@dts.upo.es
}

\begin{abstract}
RESUMEN
A menudo se ha querido ver el ámbito municipal como el más idóneo, por razones de escala, para llevar a la práctica experiencias de participación ciudadana que impliquen la democratización de la democracia representativa. A nuestro modo de ver, la escala no es, sin embargo, la única traba a que se enfrentan dichos procesos democratizadores. Existen constricciones estructurales, relacionadas con la naturaleza de los sistemas democráticos de competencia entre partidos, que determinan las preferencias de los actores sociales de forma tal que la democratización de la democracia representativa local por la vía de la participación ciudadana se hace compleja. A esta conclusión se llega al analizar, con el instrumental de la teoría de juegos, el sistema de interacción de los actores del modelo (gobierno municipal y asociaciones locales). Este sistema de interacción parece tener, además, una serie de características que guardan cierta semejanza con lo que en economía se conoce como un mercado de trastos. El modelo abstracto se aplica al estudio de dos casos concretos: el de las experiencias de participación ciudadana en los municipios de Córdoba y Málaga entre 1979 y 1995.
\end{abstract}

${ }^{1}$ Los autores quieren agradecer a Francesc Carreras, así como a un evaluador o evaluadora anónimo/a de la REIS, el haberse tomado la molestia de realizar un detallado análisis crítico del presente trabajo. Sus críticas nos han sido de gran utilidad para mejorar este artículo. Asimismo, nos han beneficiado mucho los comentarios de Miguel Beltrán, Jorge Brandt, Margarita Delgado, Juan Díez Nicolás, Antoni Domènech, Andrés de Francisco, Juan García-Bermejo, Salvador Giner, Eduardo Moyano, Manuel Pérez Yruela y Xavier Vives. Por supuesto, ninguno de ellos tiene responsabilidad en los errores que pueda tener esta versión final. Hemos de agradecerle también a Ramón Febrero la oportunidad que nos brindó de presentar este trabajo en un curso

\section{Reis}


Es un lugar común de la ciencia política que cuanto menor es el ámbito de aplicación de la democracia, más fácil resulta la participación ciudadana plena en la toma de decisiones políticas. Mientras que a escala nacional o supranacional no sería posible desarrollar una democracia con amplios cauces de participación ciudadana (una democracia participativa), ello podría darse, en cambio, en municipios pequeños o de tamaño medio (Dahl y Tufte, 1974; Sartori, 1988: 92 y ss.).

Sin embargo, a nuestro modo de ver, el problema de la «democratización de la democracia» (Parry y Moran, 1994: 11) no se reduce sólo a una cuestión de escala, sino que está relacionado también con los límites estructurales que impone a la democratización la propia democracia. Dada la naturaleza específica de nuestros sistemas políticos —-democracias representativas de competencia entre partidos-, lo que vamos a intentar mostrar aquí es que a los gobiernos municipales no les interesa promover de manera neutral y universal la participación ciudadana, y ello con independencia de las posibilidades que el ámbito local ofrezca para fomentar la participación de los ciudadanos. Se trata, pues, de tomar como unidad de análisis este ámbito, al entender que ello nos permite comprender la posibilidad real de desarrollar cauces de participación ciudadana plena y regular a pequeña escala (modelos de democracia participativa) en el seno de las democracias representativas.

Para analizar los límites de la democratización de la democracia en el ámbito local emplearemos el instrumental de la teoría de juegos. Dicha herramienta formal nos ayudará a que resulten más claros los intereses de los actores sociales que pretenden poner en marcha un proceso local de democracia participativa (gobiernos municipales y asociaciones locales, como veremos), sus estrategias y el resultado de su interacción. Así, una vez determinada en términos muy generales la naturaleza de los actores de lo que llamaremos «juego de la democratización», construimos un modelo de interacción estratégica que nos permita explicitar las limitaciones que, desde la perspectiva de los intereses del gobierno municipal, tiene todo proceso democratizador. El artículo se cierra con el estudio de dos casos a la luz de lo establecido en las secciones previas: el de las experiencias de participación ciudadana en los municipios de Córdoba y Málaga, donde durante dieciséis años (1979-1995) gobernaron sendos partidos expresamente proclives a poner en práctica instituciones de participación ciudadana que trascendieron los límites de la participación meramente electoral.

de verano de El Escorial. Por último, Fernando Aguiar quisiera agradecerle a los alumnos del curso de doctorado sobre teoría de juegos que impartió en la Facultad de Sociología de Granada durante el curso 97/98 (especialmente a Alicia, José Luis y Eugenia) sus comentarios a una primera versión de este artículo. 


\section{EL SISTEMA POLÍTICO MUNICIPAL}

Al hacer referencia al sistema político municipal en este momento de nuestro análisis, no nos referimos a un sistema político concreto en toda su complejidad, sino a los elementos mínimos más simples y abstractos compartidos por una serie de sistemas políticos municipales concretos — los de la Europa continental, como veremos en el punto siguiente-, elementos que configuran un sistema de interacción $n^{2}$. Esos elementos mínimos son los actores del modelo (gobiernos municipales y asociaciones locales, en nuestro caso), así como los recursos y acciones que controlan los actores y en los cuales están interesados (Coleman, 1990: 28 y ss.). El sistema de interacción se define, pues, por las diversas relaciones de interdependencia que establecen los actores entre sí, relaciones que dan lugar a los fenómenos sociales que queremos estudiar. En este apartado del presente artículo presentamos, por lo tanto, la naturaleza de los actores, sus recursos y sus intereses. En la siguiente sección se detallará el modelo de interacción.

\section{A) La naturaleza de los gobiernos municipales}

Si se atiende al lugar que ocupan los municipios en las distintas legislaciones constitucionales, cabe destacar dos tradiciones bien distintas. En una de ellas, más propia de la Europa continental, los municipios poseen un estatus autónomo con respecto a otros niveles de gobierno, gozando de amplias competencias para realizar las acciones de gobierno que consideren pertinentes en su ámbito. En la otra tradición, los municipios no poseen competencia propia; sólo pueden desarrollar aquellas actividades que le vengan impuestas por el parlamento, como en el caso de Gran Bretaña (ultra vires doctrine), o por los Estados, como ocurre en Estados Unidos (Dillon's Rule) ${ }^{3}$. En la primera de las tradiciones mencionadas los municipios se consideran, por tanto, como unidades autónomas de poder político, con capacidad de acción independiente de

2 Según Boudon, con quien coincidimos plenamente, pese a la enorme variedad de temas de los que se ocupa la sociología, el análisis sociológico «tiende, muy generalmente, a poner de relieve las propiedades del sistema de interacción responsable de los hechos singulares, regularidades o relaciones observados. En otros términos, los fenómenos en los cuales se interesa el sociólogo están concebidos como explicables por la estructura del sistema de interacción en cuyo interior emergen tales fenómenos» (Boudon, 1981: 36). Somos conscientes, con todo, de que el enfoque que adoptamos aquí - marcadamente deductivo - deja muchos elementos fuera del modelo. Pese a todo, no sólo ganamos de esta forma en claridad y precisión, sino que aquello que excluimos al elaborar el sistema de interacción de manera abstracta lo recuperamos, como se verá, al abordar el análisis empírico concreto.

3 Como señalan Wolman y Goldsmith, «la relación constitucional de los gobiernos locales con los gobiernos de los Estados en los Estados Unidos es muy similar a la de las autoridades locales británicas con el Estado central: los gobiernos locales no poseen un estatus autónomo» (Wolman y Goldsmith, 1992: 71). 
otros niveles de gobierno. En la segunda, el poder municipal se encuentra supeditado a niveles de gobierno superiores. Además, en el caso británico, ni siquiera existe un órgano político de carácter ejecutivo claramente separado a nivel local. En cambio, en la Europa continental sí existe un órgano ejecutivo independiente, que se refleja en la figura del Presidente o Alcalde (Francia, Italia, España, Portugal, Dinamarca) o en órganos colegiados de carácter ejecutivo (Noruega, Suecia, Alemania) (Navarro, 1998: 80 y ss.).

La existencia de un poder ejecutivo municipal autónomo tiene, además, importantes consecuencias para la política local. Por un lado, el ejecutivo puede funcionar como órgano coordinador de la acción pública municipal y posibilitar, por tanto, un mayor grado de eficacia que la que se da en los modelos políticos municipales no descentralizados (Clarke y Gaile, 1989). Por otro lado, la concepción del municipio como una entidad política independiente le otorga gran relevancia para los partidos políticos y para los ciudadanos (Blair, 1991). Para los primeros, porque el ámbito municipal puede llegar a convertirse en fiel reflejo de su poder electoral. Para los segundos, porque es el lugar hacia el que canalizan buena parte de sus demandas sociales ${ }^{4}$.

Dado que nuestro interés radica en analizar la posibilidad de que un gobierno municipal abra cauces a la participación ciudadana, nos centraremos, pues, en aquella tradición municipalista europea que otorga mayor independencia y poder a los gobiernos locales. ¿Cabe extraer algún conjunto de elementos comunes a los sistemas políticos locales autónomos que permita establecer alguna hipótesis general sobre el comportamiento de los gobiernos municipales? Antes que nada, es preciso establecer que en los sistemas políticos municipales, al igual que ocurre en el ámbito nacional, la competencia electoral entre partidos que se presentan a elecciones periódicas es el mecanismo que legitima el acceso al poder político5. A partir de aquí podemos establecer las cinco siguientes premisas; tres de ellas relativas a las limitaciones estructurales del poder del gobierno municipal y dos supuestos sobre la conducta e intereses de los actores del modelo:

1) Cada partido político constituye un equipo de personas que intentan conseguir el poder con el fin de disfrutar de ingresos, prestigio y poder o de poner en práctica su concepción del bien común.

2) El partido (o coalición) ganador tiene un control completo sobre la acción gubernamental municipal hasta la siguiente elección.

3) El gobierno local puede hacer uso de los recursos municipales (limitados) de la manera que considere más conveniente.

4 «Los problemas a los que se enfrentan los individuos en su vida cotidiana surgen en su mayor parte en el área donde viven» (Mabileau et al, 1989: 1). En este sentido, Yates (1977) señala que en el ámbito local la relación entre ciudadanos y gobierno es más intensa y cercana que en otros niveles de gobierno.

5 Una excepción a esta regla sería el caso de Suiza, donde cuentan, como es sabido, con un sistema de democracia directa en el ámbito local. Para el caso suizo, véanse Linder (1994) y Budget (1996). 
4) El gobierno local carece de competencias más allá de su municipio.

5) Todos los agentes del modelo actúan siempre racionalmente; esto es, sus preferencias son consistentes (completas y transitivas) y tratan de maximizar su beneficio.

Apoyándonos en estas premisas sobre la naturaleza abstracta del sistema local y del comportamiento de los actores en dicho sistema, podemos añadir el supuesto de que los partidos buscan siempre la forma de maximizar los votos para obtener el poder (Downs, 1973) ${ }^{6}$. Esto implica, a su vez, que el interés central del partido en el gobierno local consiste en ser reelegido. De las premisas 2 y 3 cabe derivar el hecho de que el partido (o coalición) gobernante puede hacer uso de su control del gobierno y de los recursos para favorecer su reelección.

Una vez establecidos esos cinco supuestos de partida, así como el corolario de la maximización del voto, podemos analizar las posibilidades y límites de los procesos de democractización local en el seno de la democracia representativa de competencia entre partidos. En la medida en que el gobierno municipal controla tanto los mecanismos del poder político local como los recursos, puede promover la participación ciudadana en la toma de decisiones políticas de múltiples formas. Una de ellas — que será la que analicemos nosotros por ser la más común- consiste en fomentar la existencia de asociaciones locales que representen los intereses de los ciudadanos, proporcionando a dichas asociaciones cuotas de poder. Para ello el gobierno local tiene la posibilidad de subvencionar a los grupos, ofrecerles locales de reunión, darles voz en los órganos municipales de decisión, etc. (Navarro, 1999: 61 y ss.).

Del mismo modo, un gobierno municipal no sólo puede promover la participación ciudadana fomentando la existencia de asociaciones locales mediante subvenciones y cuotas de poder, sino que también puede hacer más difícil o imposible la participación de ciertas asociaciones en los órganos de decisión. De las premisas anteriores se desprende que ese apoyo, ese fomento de la participación, está en función de la actitud de las asociaciones para con el gobierno municipal. Esa actitud bien puede ser, como veremos más abajo, de oposición o de apoyo. En otras palabras, una asociación local (o una federación de asociaciones) puede complicar, mediante la crítica al gobierno, la tarea de maxi-

${ }^{6}$ Estas cinco premisas están inspiradas en los axiomas que establece Downs (1991) sobre la naturaleza e intereses de los actores de un sistema político nacional. Aquí hemos adaptado sus axiomas al ámbito municipal y hemos transformado el primero de ellos, pues Downs no incluye entre los posibles fines que persiguen los políticos el promover su concepción del bien común. Si lo hacemos así es porque creemos que, sea cual sea el fin que persiga el político (egoísta o altruista), tendrá que maximizar sus votos para satisfacerlo - lo cual es para el político sinónimo de maximizar su beneficio-. Si yo creo sinceramente que la política X será mejor para mi ciudad que la política Y; si no me mueven sólo el dinero, el prestigio o el poder por sí solos, tendré en cualquier caso que maximizar mis votos para poder gobernar y así poner en práctica aquella política que me parece mejor. En otras palabras, suponer que los políticos tratan de maximizar una función de utilidad no significa que ésta haya de ser egoísta. 
mizar los votos, o puede favorecerla apoyando al partido o coalición gobernante. Cabe esperar que de su actitud dependerá la actuación del gobierno a la hora de decidir apoyarla o no, esto es, a la hora de otorgarle subvenciones y representación en los órganos de decisión.

Así pues, teniendo en cuenta que la democracia representativa de competencia entre partidos sólo exige que se convoquen elecciones regulares para acceder al poder, es decir, no requiere de otro mecanismo de participación ciudadana que no sea la participación electoral, ¿cuándo le resultará racional a un gobierno municipal promover la participación ciudadana? $\mathrm{O}$, dicho en otros términos, ¿cuándo serán mayores para el gobierno local los beneficios del proceso democratizador que sus posibles desventajas? Antes de dar respuesta a estas preguntas es preciso detenerse en la naturaleza del otro actor de nuestro sistema de interacción municipal: la asociaciones locales.

\section{B) La naturaleza de las asociaciones locales}

Las asociaciones locales son grupos organizados de intereses cuya finalidad es la obtención de bienes públicos locales, esto es, mejores servicios para la ciudadanía, reglamentos de participación ciudadana, mayor información sobre la marcha de los asuntos de la ciudad, etc. (el término bienes locales es de Shubik, 1992: 543). Para comprender su interacción con el gobierno municipal es necesario destacar el siguiente hecho: las asociaciones locales se ven sometidas a dos constricciones estructurales distintas: una relacionada con los rasgos característicos de la acción colectiva que emprenden; otra, con el hecho de que sólo el gobierno local puede suministrarles los bienes públicos locales que reivindican y los bienes privados que precisan (subvenciones y cuotas de poder). Veamos con detenimiento en qué consisten ambas constricciones, sobre todo la primera, pues ello nos permitirá comprender mejor la naturaleza de la interacción estratégica entre el gobierno municipal y las asociaciones locales cuando se pone en marcha un proceso de democratización, esto es, la creación de instituciones en las que las asociaciones pueden participar y tomar decisiones junto con el ejecutivo local.

Entre las distintas acciones colectivas que se pueden emprender es un hecho bien establecido que algunas se pueden llevar adelante con éxito por un pequeño grupo de activistas (la quema de una bandera, por ejemplo), mientras que en otras (huelgas, revoluciones, revueltas) el éxito no es posible sin la intervención de un gran número de personas. En el primer caso, la producción de acción colectiva está sometida a rendimientos marginales decrecientes, pues a medida que aumenta la cooperación disminuye la utilidad de nuevos apoyos individuales, hasta un punto tal en que una nueva aportación carece de efecto o incluso puede ser negativa. En el segundo caso, sometido en cambio a rendimientos marginales crecientes, la participación puede ser un incentivo para nuevas participaciones (Hardin, 1982: 17-20; Elster, 1989: 128-132; Marwell y Oliver, 1993: 40). 
El activismo local es un claro ejemplo de acción colectiva con rendimientos marginales decrecientes. Una vez superado cierto umbral mínimo de participación, la utilidad de nuevas colaboraciones disminuye considerablemente. De hecho, un pequeño grupo de activistas locales puede llevar adelante con éxito las demandas de barriadas enteras?

Así pues, en el ámbito local nos encontraremos siempre con pequeñas asociaciones de activistas muy interesados en la obtención de bienes públicos locales - para los vecinos de un barrio, para los jóvenes de la ciudad, para las mujeres... - que han de hacer frente al problema del gorrón (free rider), al menos hasta que superan un umbral mínimo de participación. Para superar ese umbral precisan, en gran medida, de los recursos que les pueda proporcionar el gobierno local (subvenciones, locales, bienes organizativos o puestos en los órganos locales de decisión).

Pero es que, además, como vimos antes, los bienes públicos que desean obtener dichas asociaciones sólo los proporciona el gobierno local. Todo ello implica que la existencia y actividad de los mismos depende en gran medida de la actitud del equipo municipal de gobierno. Sin embargo, esa dependencia se halla lejos de ser absoluta: en primer lugar, porque las asociaciones también pueden amenazar al partido (o coalición) en el gobierno municipal con una actitud de permanente crítica que dificulte la tarea de maximizar los votos. Habrá asociaciones dispuestas a colaborar con el gobierno local, asociaciones que pueden ser ideológicamente afines (o al menos neutrales) y asociaciones contrarias al gobierno. Así pues, dada la naturaleza de las asociaciones locales, su interés se centra en conseguir el apoyo máximo del equipo municipal de gobierno: las asociaciones afines ofrecerán su apoyo a cambio de que el gobierno local las apoye a su vez, y las adversas buscarán un cambio de gobierno que dé lugar a una nueva mayoría que les resulte favorable.

\section{GOBIERNO MUNICIPAL Y ASOCIACIONES LOCALES: EL JUEGO DE LA DEMOCRATIZACIÓN}

¿Cuál puede ser, pues, el resultado de la interacción entre un gobierno municipal interesado en abrir cauces a la participación ciudadana y las asociaciones locales, dados sus respectivos intereses? Aunque un gobierno municipal esté interesado en democratizar la democracia local —al menos en su ámbito de competencia-, ello ha de ser compatible para él con la lógica de la demo-

Como señala Oliver (1984: 602), «el activismo local muestra a menudo esta propiedad de los rendimientos marginales decrecientes. Thursz [1972] ha demostrado que las organizaciones locales que tienen éxito no precisan de la participación de masas, y cita la afirmación de Alinsky de que la participación de un 3 por 100 de una comunidad aseguraría el éxito de una organización local. Bolduc [1980] realiza un típico estudio de caso de una barriada en la que sólo una docena de residentes participaban activamente en la organización vecinal, si bien era considerada como legítimamente representativa por la mayoría de los residentes». 
cracia representativa: en el momento de las elecciones debe obtener el mayor número de votos posibles para ser reelegido. Así pues, de lo dicho en la sección anterior se desprende que, de alguna forma, el interés por democratizar la democracia haciendo partícipes en la toma de decisiones a las asociaciones locales representativas deberá supeditarse al interés por maximizar el voto. A su vez, las asociaciones locales tratarán de beneficiarse de los recursos que el gobierno municipal pueda poner a su disposición, sin dejar por ello de defender los intereses de los ciudadanos a los que representan; intereses que pueden estar abiertamente enfrentados a los del partido o coalición en el gobierno (ya sea por motivos ideológicos o por cualesquiera otros).

Teniendo en cuenta lo que acabamos de afirmar, para comprender la interacción entre el gobierno municipal y las asociaciones locales será preciso establecer primero en qué contexto informativo se desenvuelve esa interacción. Desde el punto de vista de la información con que cuenta el gobierno municipal sobre el tipo de asociaciones locales que participarán en el proceso democratizador, el contexto puede ser de certidumbre (el gobierno tiene información perfecta sobre la naturaleza de las asociaciones de su municipio) o de incertidumbre (el gobierno tiene información imperfecta) ${ }^{8}$. Si el gobierno municipal, que lleva la iniciativa en el «juego de la democratización», tiene plena certeza de hallarse ante asociaciones afines, optará por la democratización, abriendo cauces a la participación en los órganos municipales de decisión y subvencionando a las asociaciones locales. Si, por el contrario, el gobierno tiene la certeza de que las asociaciones locales le son adversas —esto es, su estrategia no es cooperativa-, no las fomentará, y se paralizará el proceso de democratización. Cuando el gobierno municipal sabe que la trama asociativa de su municipio está configurada por asociaciones locales críticas, contrarias a su política, le resultará difícil conciliar su interés por la democratización y por maximizar el voto: la actitud crítica de las asociaciones locales representativas puede restarle votos, más aún si el gobierno municipal les otorga voz en los

${ }^{8}$ Siguiendo a F. Knight, que estableció la distinción en su obra Risk, Uncertainty and Profit (1921), la teoría de la decisión diferencia claramente entre riesgo e incertidumbre. Nos encontramos en una situación de riesgo cuando no existe certeza sobre el resultado de la decisión, aunque se conoce al menos la probabilidad objetiva de los distintos resultados alternativos. Éste sería el caso, por ejemplo, de la elección entre cara y cruz de una moneda: desconocemos de antemano el resultado (si la moneda no está trucada, claro está), pero conocemos la probabilidad objetiva de las dos alternativas. Las situaciones de incertidumbre se caracterizarían, en cambio, por el hecho de que no sólo desconocemos a priori el resultado final, sino que no podemos predecirlo tampoco en términos de probabilidades objetivas: sólo cabe atribuir probabilidades subjetivas a las consecuencias de nuestra acción. A nuestro modo de ver, el gobierno municipal que desconoce la composición de la trama asociativa de su municipio se enfrenta a una situación de incertidumbre, no de riesgo. En contextos estratégicos la incertidumbre implica que los jugadores tienen información imperfecta: sus respectivos conjuntos de información tienen más de un elemento (esto es, varios de los nodos que unen las distintas ramas de un juego en su forma estratégica se encuentran en un mismo conjunto). Si, además, uno de los jugadores posee información que el otro no posee, como es el caso de nuestro juego de la democratización, la información es asimétrica. Sobre juegos e información, véase Rasmusen (1989), cuyas definiciones seguimos aquí. 
órganos políticos de decisión. Ante una situación así, el gobierno preferirá no crear mecanismos democratizadores.

En un contexto de certidumbre puede ocurrir también que el gobierno sepa cuáles son las asociaciones afines y cuáles no, fomente a las primeras y no a las segundas (o fomente mucho más a las primeras) y se produzca una democratización parcial, una apertura sesgada de los órganos de poder local en la que las asociaciones adversas quedan parcial o totalmente excluidas.

Sin embargo, no siempre un gobierno municipal tiene información perfecta sobre la trama asociativa de su municipio, o bien obtiene la información de que carece cuando ya lleva cierto tiempo en el poder y ha tomado la decisión de fomentar la participación. Hemos de pensar que en ciudades de tamaño medio (no digamos ya si son grandes) puede haber cientos o miles de asociaciones locales (de vecinos, de consumidores, de jóvenes, ecologistas, de mujeres, de la tercera edad, etc.). Ya sea, pues, porque se trata de un gobierno nuevo que desconoce aún el contexto social en que se desenvuelve, ya sea porque el número de asociaciones es elevado o porque surgen asociaciones nuevas de difícil catalogación, el ejecutivo local puede hallarse en una situación de información imperfecta que le haga dudar de la conveniencia —en términos de su interés central, esto es, maximizar el voto- de la democratización. En tal caso, el gobierno desconoce el tipo de asociación a la que decide apoyar, afín o contraria, y corre el peligro de equivocarse — desde el punto de vista de sus intereses, insistimos - en su decisión. En tal situación de incertidumbre, ¿cuándo resultará racional para el gobierno crear órganos políticos locales democratizadores, órganos en los que participen las asociaciones locales representativas de los intereses ciudadanos? En ese caso, en un contexto de incertidumbre, el gobierno municipal podrá, como mucho, estimar subjetivamente la probabilidad de que unas asociaciones sean afines y otras contrarias a su política, y actuará en función de su creencia (figura 1).

Como refleja la figura 1, el gobierno municipal (GM) se enfrenta a la decisión inicial de democratizar (D) o no (ND) la democracia local (suponiendo siempre, como venimos haciendo, que tenga esa potestad). Así pues, para que se pueda dar el juego de la democratización partimos del supuesto de que el gobierno prefiere, en primer lugar, democratizar el muncipio, pero ello siempre y cuando crea que las asociaciones van a ser cooperativas, que no le resultarán adversas. Si es así, espera obtener un resultado de b-c, es decir, el beneficio que obtiene al abrir cauces de participación ciudadana en un contexto en el que las asociaciones locales apoyan su política (estrategia A de las asociaciones locales) menos los costes inevitables de fomentar a las asociaciones?. De otra forma preferirá no democratizar; preferirá dejar las cosas como están $(0,0)$,

9 El beneficio no sólo es político, sino que también podría ser económico, en la medida en que las asociaciones puedan participar en la financiación de proyectos municipales. El coste está relacionado con las subvenciones a las asociaciones, la cesión de cuotas de poder, la cesión de locales, etc. Esto se verá mejor al estudiar los casos de Córdoba y Málaga. 


\section{FIGURA 1}

\section{Juego de la democratización municipal}

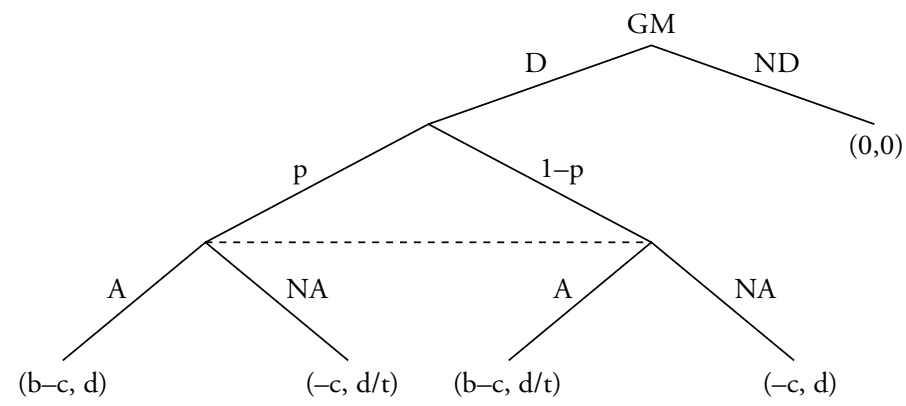

b =beneficio para el gobierno municipal (GM) de democratizar (D) y que las asociaciones le den su respaldo político;

$-\mathrm{c}=$ coste de apoyar el proceso democratizador (subvenciones a asociaciones, precio de locales cedidos, cesión cuotas de poder, etc.);

$\mathrm{d}$ =beneficio para las asociaciones de que el GM las fomente (subvenciones, locales, poder, etc.);

$1 / \mathrm{t}=$ factor de corrección del beneficio de las asociaciones por no apoyar (NA) a un gobierno afín o por apoyar (A) a uno adverso ${ }^{10}$;

p =probabilidad de que la asociación sea afín; siendo, por lo tanto, 1-p la probabilidad de que sea adversa.

Orden de preferencias del gobierno municipal: $\mathrm{b}-\mathrm{c}>0>-\mathrm{c}$.

Orden de preferencias de las asociaciones: $\mathrm{d}>\mathrm{d} / \mathrm{t}>0$.

pues teme que la democratización sólo le acarree costes (lo que se refleja en el resultado negativo -c). El GM cree, además, que si se halla en un contexto de asociaciones afines, éstas apoyarán su política, elegirán a buen seguro la estrategia $\mathrm{A}$; mientras que si se halla ante una trama asociativa adversa, las asociaciones elegirán la estrategia de no apoyar (NA) la política del GM. ¿Cuándo le resultará racional, entonces, democratizar la democracia local? Dado que se halla en una situación de incertidumbre (reflejada en la figura 1 por el movimiento probabilístico que se da en el juego una vez que el GM decide democratizar), un gobierno municipal que trate de maximizar su beneficio sólo democratizará cuando ocurra que $\mathrm{pb}>\mathrm{c}$. En otras palabras, para el gobierno

${ }^{10}$ La asociación contraria al gobierno municipal que apoye su política ha de valorar el coste de ese apoyo, el cual podría verse reflejado, por ejemplo, en la pérdida de asociados y, por lo tanto, de fondos. Y otro tanto le ocurre a las asociaciones afines. Esa valoración queda reflejada en el juego al considerar que el posible beneficio de apoyar al gobierno (las adversas) o no apoyarlo (las afines) ha de ser corregido por el número de socios que creen que pueden perder con su estrategia $(1 / 2,1 / 3,1 / 4$; es decir, $1 / \mathrm{t})$. 
municipal resultará rentable la democratización cuando la probablidad subjetiva de que la trama asociativa sea afín, multiplicada por el beneficio de la democratización, sea mayor que el coste de la misma. En la medida en que el GM crea que p se acerca a 0 , tendrá un fuerte incentivo para no democratizar la política local ${ }^{11}$. Por otra parte, aunque el GM crea que p se aproxima a 1 , si considera que el beneficio de la democratización es muy pequeño (de forma que el coste incluso de apoyar a asociaciones afines sea grande), tampoco tendrá motivos para democratrizar.

Así pues, nuestra hipótesis es que incluso para un gobierno local interesado en promover la participación ciudadana más allá de la mera participación electoral, si se halla en una situación de información imperfecta, sólo le resultará racional promover dicha participación en caso de que crea que $\mathrm{pb}>\mathrm{c}$.

Es interesante comprobar que el resultado obtenido hace que el juego de la democratización municipal se asemeje formalmente a lo que se conoce en economía como "mercado de trastos» (market for lemons). En el mercado de trastos (por ejemplo, un mercado de coches de segunda mano) el comprador desconoce la calidad de aquello que va a comprar y no tiene forma de averiguarlo. Por este motivo, si el comprador sospecha que los productos del vendedor son de mala calidad — son trastos—, no comprará nada. La incertidumbre sobre la calidad de los bienes puede producir una quiebra del mercado. Sólo la confianza del comprador en los vendedores (que se puede establecer mediante una garantía, por ejemplo) permite que el mercado funcione (Akerlof, 1970).

Pues bien, de nuestro modelo parece desprenderse que los gobiernos municipales actuarían frente a las asociaciones como lo haría un comprador en un mercado de trastos: trata de reducir la incertidumbre intentando averiguar la calidad de las asociaciones y procurando excluir del mercado político a los «trastos». Ahora bien, mientras que la presencia de «trastos» es muy perjudicial para el mercado de bienes, pues su mala calidad es algo objetivo, la calidad del «trasto» político es subjetiva —el gobierno decide si son, para él y por motivos ideológicos, «trasto» o no-, y su exclusión implica una limitación del proceso de democratización de la democracia ${ }^{12}$.

Del mismo modo, pues, que el comprador de un bien ha de tener confianza en que el vendedor no le está engañando y vendiendo un mal producto, el

${ }_{11}$ Mediante un proceso de inducción inversa —es decir, analizando el juego desde el final一, el GM supone que las asociaciones locales afines le apoyarán y, por tanto, obtendrá una utilidad esperada de ese apoyo de $\mathrm{p}(\mathrm{b}-\mathrm{c})$; y supone también que las adversas no le apoyarán, por lo que la utilidad esperada será de $-\mathrm{c}(1-\mathrm{p})$. Con una sencilla operación se obtiene $\mathrm{pb}>\mathrm{c}$. Es cierto que las asociaciones podrían enviar alguna señal que permitiera al GM reconocer de qué tipo son. Esto permitiría analizar el proceso de democratización como un juego de señales. La ideología de las asociaciones, por ejemplo, sería una forma de señal que reduciría la incertidumbre. Sin embargo, la diversidad de asociaciones suele ser tal que la emisión de señales resultaría inutil, las asociaciones podrían fácilmente confundir al GM, y tendríamos lo que se conoce como un cheap-talk game, es decir, un juego de parloteo en el que el valor de la señal es nulo.

${ }_{12}$ Excepto en el caso en que las asociaciones excluidas sean abiertamente hostiles a la democracia misma en cualquiera de sus formas. 
gobierno municipal pretende apoyar su proceso de apertura democrática en las asociaciones locales en las que confía. La quiebra de la confianza entre comprador y vendedor produciría un fallo del mercado de bienes; la quiebra de la confianza entre asociaciones y gobierno municipal provocaría el fracaso del proceso democratizador. Ahora bien, mientras que la relación de confianza entre el comprador y el vendedor conduce al éxito total del mercado, pues el vendedor coloca sus mercancías en el merado y el comprador obtiene bienes de buena calidad (Akerlof; 1970; Dasgupta, 1988; Gardner, 1996: 276), la confianza entre el gobierno municipal y las asociaciones locales sólo conduce a una apertura limitada del proceso democratizador, pues las asociaciones no afines al gobierno, en las que éste no confía políticamente, pueden quedar excluidas de dicho proceso. Desde el punto de vista de una concepción normativa de la democracia participativa sería preciso, pues, que el gobierno municipal realmente interesado en democratizar la democracia no actuara como si fuera un comprador en un mercado de trastos, y abriera cauces de participación a todas las asociaciones democráticas, afines o no. Ahora bien, ¿en qué medida es posible llevar esto a cabo en el seno de la democracia representativa de competencia entre partidos?; ¿̇en qué medida puede surgir la democracia participativa del seno de la democracia representativa sin que esta última sufra importantes transformaciones? Aunque responder a estas preguntas nos llevaría mucho más allá del objeto de nuestro artículo, el modelo que hemos elaborado pretende evidenciar las dificultades que entraña esa tarea; dificultades ilustradas empíricamente, en el apartado que sigue, mediante el análisis de las experiencias de participación de asociaciones locales en los órganos municipales de decisión de los Ayuntamientos de Córdoba y Málaga.

\section{DOS ESTUDIOS DE CASO: GOBIERNO MUNICIPAL Y ASOCIACIONES LOCALES EN LOS AYUNTAMIENTOS DE CÓRDOBA Y MÁLAGA ${ }^{13}$}

De la hipótesis que defendemos aquí se desprende, pues, que si un gobierno municipal interesado en abrir cauces a la participación ciudadana actúa de manera racional, cuando se halle en un contexto de certidumbre tratará de no fomentar de manera equitativa a las asociaciones locales representativas de los intereses ciudadanos, sino que lo hará de forma sesgada, intentando favorecer a los grupos que le apoyan. Y si el contexto es de incertidumbre sólo le resultará racional la democratización si cree que la probabilidad subjetiva de hallarse ante asociaciones afines es lo suficientemente grande como para que el beneficio sea mayor que el coste de la democratización. ¿Podemos ilustrar esto con algún caso real? Podemos ilustrarlo, en efecto, tanto para el caso del Ayuntamiento de Córdoba como para el de Málaga. En ambas ciudades se produjo

${ }^{13}$ La información empírica para elaborar esta sección procede de Navarro $(1998,1999)$. 
una intensa relación entre asociaciones locales y gobierno municipal desde las primeras elecciones locales democráticas de 1979, y en ambas ciudades, además, gobernaron de manera ininterrumpida desde 1979 hasta 1995 partidos políticos programáticamente interesados en promover la participación ciudadana (PCE/IU en Córdoba y PSOE en Málaga) ${ }^{14}$.

En el municipio de Córdoba la participación ciudadana se articula fundamentalmente a través de dos tipos de órganos, el Consejo de Distrito y el Consejo de Centro. Según el Reglamento de Participación Ciudadana (RPC) de esta ciudad la composición del Consejo de Distrito es la siguiente:

1) Un representante de cada una de las Asociaciones de Vecinos del distrito.

2) Un representante del Consejo Local de la Juventud.

3) Un representante de las Asociaciones de Padres de Alumnos que lo hayan solicitado a través de su Federación.

4) Un representante de los grupos culturales que lo hayan solicitado a través de su Federación.

5) Un representante de cada una de las organizaciones legalmente constituidas y que lo hayan solicitado formalmente al actual Consejo de Distrito, procurando que estén representadas todas las sensibilidades.

6) Un representante del Ayuntamiento.

Cada distrito debe contar con un Centro Cívico Municipal, cuyo Consejo es elegido por el Pleno Municipal, a propuesta de los Consejos de Distrito. Los Centros Cívicos, que son uno de los principales cauces de participación ciudadana en Córdoba, cuentan con un Consejo constituido por un Presidente el Director del Centro-, un Secretario, tres técnicos municipales y seis representantes vecinales.

Pues bien, estos dos órganos, a través de los cuales se encauza la participación ciudadana, estuvieron prácticamente copados por las Asociaciones de Vecinos (AA.VV.) entre 1979 y 1995 (que es el período en que, como hemos dicho, se centra nuestro análisis). La presencia de estas asociaciones en comparación con la de otros grupos era considerablemente mayor: de un total de 85 miembros de los siete Consejos de Distrito, 47 pertenecen a AA.VV; y de un total de 30 miembros de los cinco Consejos de Centro existentes, 23 son miembros a su vez de Asociaciones de Vecinos.

El mismo RPC, que rige la composición de los Consejos de Distrito, favorece considerablemente a las AA.VV., pues mientras que éstas deben contar con al menos un representante de todas las asociaciones del distrito, el resto de

${ }^{14} \mathrm{El}$ análisis de documentos municipales muestra que en ambos casos se tiene como objetivo del programa de gobierno potenciar la participación ciudadana, y ello con anterioridad a la existencia de algún tipo de legislación que regulase este asunto. Una vez aparecida tal legislación - la Ley Reguladora de Bases de Régimen Local de abril del 85-, estos municipios han desarrollado, además, procesos que no eran de carácter obligatorio (aunque estaban recogidos en la Ley) y otros que han dependido de su propia iniciativa más allá del marco normativo vigente. 
grupos tienen que solicitarlo. Ello supone que, en lo que respecta a la presencia de asociaciones en los Consejos de Distrito de Córdoba, la distribución es claramente desigual. Y otro tanto ocurre en los Centros Cívicos, donde la probabilidad de presencia de representantes vecinales $\left(\mathrm{T}_{\mathrm{i}}\right)$ resulta aún más patente (tabla 1) ${ }^{15}$. Pues si en el caso de los Consejos de Distrito la probabilidad de que un miembro del Consejo Tipo (construido a partir de la media de los siete existentes) sea a su vez miembro de una Asociación de Vecinos es de 0,5, en el caso de los Consejos de Centro ésta aumenta a 0,66, desapareciendo, además, la representación de la mayoría de los grupos.

\section{TABLA 1}

Composición de Consejo de Distrito y Consejo de Centro: tasa de presencia e indice de representación en Consejos Tipo

\begin{tabular}{|c|c|c|c|c|}
\hline \multirow[b]{2}{*}{ Tipo de asociación } & \multicolumn{2}{|c|}{ Consejo de Distrito } & \multicolumn{2}{|c|}{ Consejo de Centro } \\
\hline & $T p_{i}$ & $I R_{i}$ & $T p_{i}$ & $I R_{i}$ \\
\hline 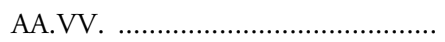 & 50,00 & 29,23 & 66,00 & 45,23 \\
\hline 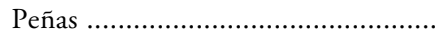 & 8,33 & $-10,13$ & 0,00 & $-18,46$ \\
\hline APA & 8,33 & $-12,70$ & 0,00 & $-21,03$ \\
\hline  & 8,33 & $-8,08$ & 16,00 & $-0,41$ \\
\hline 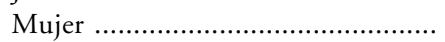 & 8,33 & 5,26 & 16,00 & 12,93 \\
\hline Tercera Edad ................................. & 8,33 & 5,77 & 0,00 & $-5,77$ \\
\hline 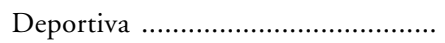 & 8,33 & $-9,37$ & 0,00 & $-9,77$ \\
\hline \multicolumn{5}{|l|}{ 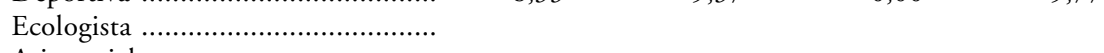 } \\
\hline \multicolumn{5}{|l|}{ 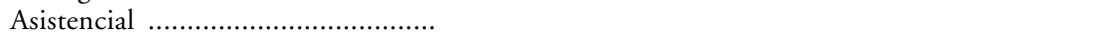 } \\
\hline TOTAL & 100,00 & 0,00 & 100,00 & 0,00 \\
\hline
\end{tabular}

FUENTE: Navarro (1998: 136).

- $\mathrm{Tp}_{\mathrm{i}}$ : Tasa de presencia del grupo i (porcentaje de miembros de cada tipo de asociación sobre el total de cada Consejo).

- IR: Índice de representación (Tpi menos peso de cada tipo de asociación sobre el total de asociaciones en el municipio).

El especial fomento de las AA.VV. por parte del gobierno municipal resulta evidente de nuevo al analizar las subvenciones concedidas por el Ayuntamiento

15 Para calcular la tasa de presencia $\left(\mathrm{Tp}_{\mathrm{i}}\right)$ de cada tipo de asociación se ha construido un Consejo tipo. Para ello se calcula el número medio de miembros que posee cada tipo de asociación en todos los Consejos de Distrito o de Centro. La tasa de presencia en el Consejo tipo es el porcentaje que supone este número medio de miembros de cada tipo de asociación sobre el total de los miembros del Consejo tipo. 
a las asociaciones locales. En este caso, como se aprecia en la figura 2 — donde se representa el Índice de Acceso a Subvenciones (IDAS) —, las AA.VV. reciben un porcentaje de la cantidad total destinada a subvenciones superior al porcentaje que representan sobre el total de colectivos subvencionados $^{16}$.

\section{FIGURA 2}

\section{Indice de Acceso a Subvenciones de centros cívicos en el municipio de Córdoba} (1993-1995)

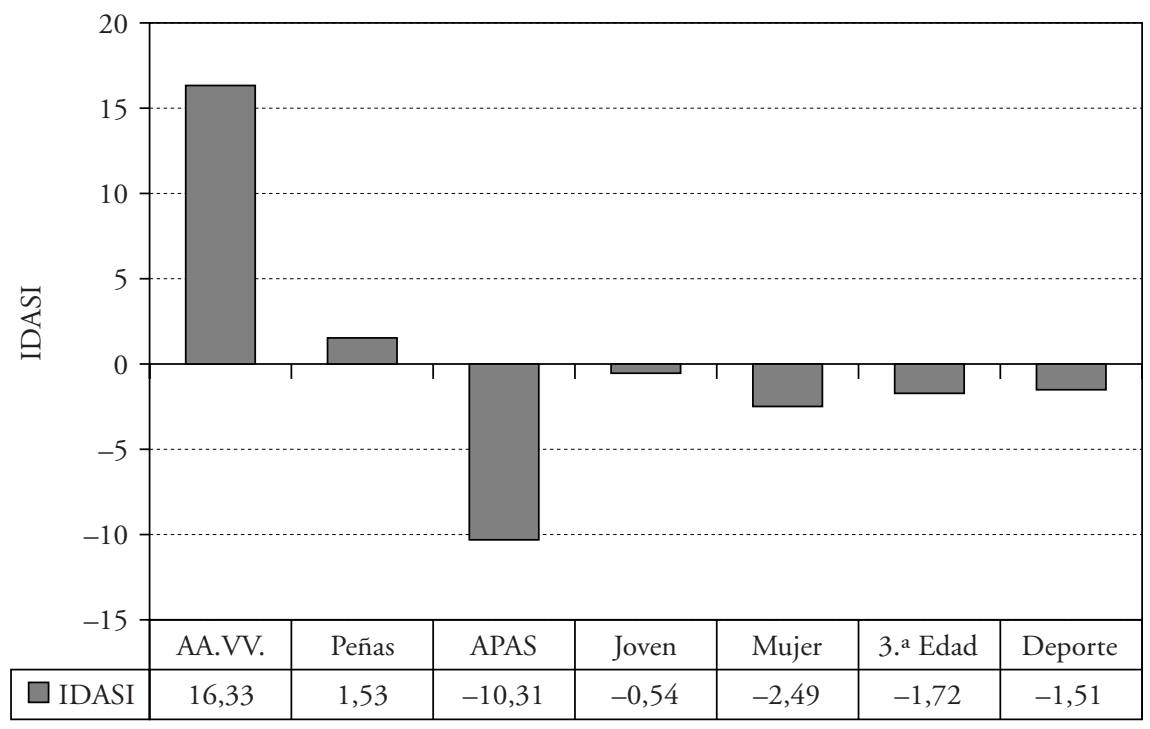

Tipo de colectivos

FUENTE: Navarro (1999: 237).

¿Supone todo esto, acaso, que el gobierno municipal fomenta en especial a aquellas asociaciones que cree que le favorecen más? Podría ser que no. Podría ser que en el caso del municipio de Córdoba existiera un número mucho mayor de asociaciones de vecinos que de otras asociaciones locales. En ese

${ }^{16}$ El IDAS mide la desigualdad en la distribución de las subvenciones. Se calcula de la forma siguiente: $\operatorname{IDAS}_{\mathrm{i}}=\mathrm{pcs}_{\mathrm{i}}-\mathrm{ps}_{\mathrm{i}}$, donde:

- pcs: proporción que supone la cantidad que recibe cada tipo de asociación sobre el total;

- ps: proporción que supone el número de asociaciones subvencionadas de cada tipo sobre el total de las subvencionadas. 
caso, la distribución de subvenciones y la presencia en los órganos de participación no sería desigual, sino que sería proporcional al número de grupos locales existentes. Sin embargo, esto no es así. De las 390 asociaciones locales existentes en Córdoba, las AA.VV. representan un 20,77 por 100 del total (Navarro, 1998: 138). A pesar de que se trata de un porcentaje considerablemente elevado, la cantidad de subvenciones que reciben estas asociaciones resulta muy superior, en términos relativos, a su número. Por lo que se refiere a los Consejos de Distrito y Consejos de Centro, el Índice de Representación (IR $)$ - calculado como diferencia entre la tasa de presencia de cada grupo y su peso sobre el total de la trama asociativa local- muestra claramente una mayor representación de las AA.VV. (tabla 1).

A nuestro entender, el hecho de que la corporación municipal cordobesa tuviera información perfecta sobre las Asociaciones de Vecinos y supiera, por tanto, que, frente al resto, le eran claramente afines es lo que explica el especial fomento de aquéllas. Aun manteniendo su independencia de criterio (pues la estrategia de las Asociaciones de Vecinos no es de apoyo incondicional), las AA.VV. estuvieron ligadas desde su origen a la órbita del Partido Comunista y de otros partidos de izquierda. Esta interrelación fue diluyéndose, sin embargo, a medida que se afianzaba la democracia en España: muchos de los partidos de izquierda más activos desaparecieron, el PCE perdió en las urnas buena parte de su fuerza e influencia y el movimiento vecinal se fue despolitizando gradualmente (Amodio, 1986; Castell, 1986: 318-319; Gunther y otros, 1986: 176).

Ahora bien, la permanencia del PCE —integrado a partir de 1985 en la coalición Izquierda Unida, de la que es su motor principal- en la alcaldía de Córdoba desde las elecciones municipales de 1979 hasta las elecciones del año 95, no sólo permitió que aquella relación privilegiada entre el PCE y el movimiento vecinal no desapareciera, sino que las AA.VV. encontraron en el poder municipal mayor fomento que otras asociaciones locales. Tal y como apunta el antiguo Secretario de la Federación Cordobesa de Asociaciones de Vecinos, «la mayoría de las AA.VV. están nutridas por gente de IU y por cristianos progresistas». El gobierno municipal de Córdoba, si bien no tiene información perfecta sobre la trama asociativa de la ciudad en su totalidad, sí la tiene sobre las AA.VV. Sabe que un cuarto del total de asociaciones de la ciudad son de vecinos y tiene la seguridad de que apoyarán su política si las fomenta — tiene la seguridad de que son afines-, y en ellas sustenta, como vemos, el desarrollo de instituciones de participación ciudadana.

Antes de decidir si fomentará o no la participación, o si privilegiará en mayor medida a unas asociaciones en lugar de otras, el partido o coalición en el gobierno municipal se trazará, pues, un mapa aproximado de la información que tiene sobre las asociaciones locales. En este sentido resultan muy aclaradoras las palabras del concejal del PSOE que desde principios de los ochenta fue responsable del área de participación ciudadana del Ayuntamiento de Málaga: 
«El colectivo de jubilados... depende de quién le dé. Fundamentalmente en las clases más modestas derivan todos hacia el PSOE. El colectivo de jóvenes... Dios supiera, Dios supiera. Las culturales... depende. Las mujeres... son progresistas. Pero normalmente el movimiento es mucho más radicalizado.»

A tenor de ese conocimiento, el gobierno municipal decidirá si fomenta a las asociaciones o a cuáles fomenta. Precisamente el caso del Ayuntamiento de Málaga nos permite ilustrar la estrategia de un gobierno municipal que cree que las asociaciones más numerosas de la ciudad y que con más representación cuentan en los órganos locales de decisión, las de vecinos, le son adversas.

Entre 1979 y 1995 el gobierno municipal de Málaga pasa por dos estapas bien diferenciadas. La primera de ellas transcurre desde el año 1979 hasta 1983, y se caracteriza por gobernar la ciudad una coalición del PCE y del PSOE. La segunda, desde el 84 al 95, supone el gobierno en solitario del PSOE. En aquella primera etapa de gobierno en coalición el ejecutivo municipal se apoya en las AA.VV. para poner en marcha el primer Reglamento de Participación Ciudadana. Este primer Reglamento es el intrumento diseñado por el gobierno municipal y las AA.VV. para desarrollar un modelo de democracia participativa que recogiera la siguiente división del trabajo: los representantes políticos, elegidos en elecciones periódicas, toman las decisiones y los representantes de las asociaciones canalizan hacia los políticos las demandas de los ciudadanos a través de los órganos de participación (en el caso de Málaga, el Pleno de Distrito). Esa división del trabajo queda plasmada en el Reglamento de Participación Ciudadana, que en su primera versión concede un gran peso, como vemos en la tabla 2, a las AA.VV.

Con anterioridad a 1983 (año en que se convocan por segunda vez elecciones municipales y el PSOE obtiene en Málaga la mayoría absoluta), la afinidad que existe entre las AA.VV. y uno de los miembros de la coalición de gobierno, el PCE, permite — como en el caso de Córdoba - que la apertura de cauces de participación se realice favoreciendo a las asociaciones vecinales ${ }^{17}$. Sin embargo, el triunfo del PSOE en 1983 por mayoría absoluta obliga a este partido a plantearse una nueva estrategia: o bien desactivar la participación ciudadana (la estrategia ND del juego de la sección anterior), lo que quedó descartado, o bien buscarse nuevos aliados frente a las AA.VV. ${ }^{18}$. Y ello porque se tiene la

17 En el momento en que se redacta y aprueba el primer Reglamento, el Concejal responsable de participación — acción vecinal— es del PCE, y los miembros de la Federación de AA.VV. son cercanos a este partido. Este Concejal señala lo siguiente: «Entonces se elaboraron los primeros documentos de participación. Que me encargo yo de elaborar junto con la Federación de AA.VV., que también lógicamente al frente de la Federación había otro del Partido Comunista, que..., que esto era así entonces».

${ }_{18}$ Frente a los miembros del PSOE que siguen apostando por la participación, otros, en cambio, creen que las elecciones los legitiman para gobernar la ciudad sin contar con la opinión de las asociaciones locales: "Pero bueno, vuelvo a decir que tengo compañeros que, con todo el respeto que se merecen, que decían: "Hemos ganado las elecciones"... Aquí ha habido compañe- 


\section{TABLA 2}

Composición de los Plenos de Distrito en el municipio de Málaga (1981-1995)

\begin{tabular}{|c|c|}
\hline Reglamento & Pleno de Distrito \\
\hline $\begin{array}{l}1981 \\
1983\end{array}$ & $\begin{array}{l}\text { Presidente: Concejal nombrado por el Alcalde } \\
\text { Vocales: por cada grupo político en proporción al Pleno } \\
1 \text { por cada Asociación de Vecinos } \\
1 \text { de la Federación de AA.VV. } \\
1 \text { representante de todas las APAS } \\
3 \text { de entre el resto de asociaciones } \\
\text { (RPC } 79 / \mathrm{II} / 3 \text { y } 4)\end{array}$ \\
\hline $\begin{array}{l}1984 \\
1988\end{array}$ & $\begin{array}{l}\text { Presidente: Concejal nombrado por el Alcalde } \\
\text { Vocales: por cada grupo político en propoción al Pleno } \\
1 \text { de cada una de las AA.VV. } \\
1 \text { de la Federación de AA.VV. } \\
2 \text { de las APAS } \\
1 \text { de la Federación de APAS } \\
1 \text { por cada uno de las otras asociaciones } \\
\text { (RPC } 84 \text { y } 88 / \mathrm{III} / 3 \text { ) }\end{array}$ \\
\hline 1995 & $\begin{array}{l}\text { Presidente: Concejal nombrado por el Alcalde } \\
\text { Vocales: por cada grupo político en propoción al Pleno } \\
1 \text { por cada Asociación de Vecinos } \\
1 \text { de la Federación de AA.VV. } \\
2 \text { de las APAS } \\
1 \text { de la Federación de APAS } \\
1 \text { de la Federación de Peñas } \\
2 \text { por cada uno de los otros tipos de colectivos } \\
\text { (RPC } 95 / \mathrm{III} / 5 \text { ) }\end{array}$ \\
\hline
\end{tabular}

FUENTE: Navarro (1998: 141).

convicción de que el aliado principal de la coalición que gobernó entre 1979 y 1983, las asociaciones vecinales, no es afín a la nueva mayoría y no cabe otorgarle el apoyo que se le venía concediendo:

«El movimiento asociativo de vecinos, su creación no es del PCE, pero el PCE lo convierte en arma suya. Cuando comienzan las primeras elecciones, fue su caballo de batalla... y por eso, pues pretenden manipular todo lo que sea colectivos asociativos, fundamentalmente centrándose en las AA.VV.» (Concejal del PSOE responsable del área de participación).

ros que... unas elecciones y se ríen de ti [por fomentar la participación]. “¿Y qué hacen estos tíos [representantes de asociaciones locales] opinando de [las decisiones políticas]?”" (Concejal del PSOE responsable del área de participación ciudadana). 
Con independencia de que ello sea cierto o no, el caso es que el partido en el gobierno municipal así lo cree, y por ello intenta contrarrestar la capacidad de influencia del movimiento vecinal mediante una reforma del Reglamento de Participación que dé cabida a nuevos grupos ${ }^{19}$. En concreto, el PSOE trata de dar mayor representatividad en los Plenos de Distrito a las Asociaciones de Padres de Alumnos (APAS), por considerarlas más afines políticamente:

«Las APAS tenían una Federación muy del PSOE... Cuando empecé a movilizar a los movimientos sectoriales fui colegio por colegio fomentando la existencia de APAS, como yo decía, creando al enemigo» (Concejal del PSOE responsable de participación ciudadana; cursiva nuestra).

La oposición de las asociaciones vecinales a la reforma del Reglamento ${ }^{20}$ y la debilidad de las APAS como aliado (en la medida en que la educación no es una competencia municipal) hacen que la estrategia del PSOE no tenga pleno éxito. Como se ha visto en la tabla 2, las sucesivas reformas del RPC, si bien no merman la representatividad de las AA.VV., al menos intentan diluirla con una mayor presencia de representantes de asociaciones más afines al gobierno municipal (o, dicho en los términos de nuestro modelo, lo que trata el PSOE es de incrementar p). Sin embargo, en la figura 3 se aprecia, mediante el IR, que a mediados de los noventa las AA.VV. siguen estando sobrerrepresentadas en relación con las demás asociaciones locales ${ }^{21}$.

19 Sobre la conveniencia o no de fomentar a partir del 83 la participación ciudadana, un responsable del PSOE nos dice: "Yo recuerdo que aquí quisimos mover el tema de las asociaciones y de las subvenciones, pero hace ya años. “¿Qué queréis, poner un sueldo a los del PCE?”. Ésa fue la contestación oficial del PSOE, te estoy hablando del PSOE... "Entonces, ¿[es] que le queréis poner un sueldo a los liberados?, ¿queréis liberar a la gente del PCE?”. Era una actitud muy razonable porque era verdad».

${ }^{20}$ «Al principio la primera batalla que se libró fue que la Federación de Vecinos quería más o menos la exclusiva» (Concejal del PSOE del área de participación ciudadana). En opinión de las AA.VV., el PSOE trata de limitar su papel mediante la reforma del RPC, a lo cual se oponen: «... revisando el documento [el nuevo RPC] pues nos tiramos un año, donde veíamos cómo todo aquel espíritu del primer documento que había nacido propiciado por el movimiento vecinal de Málaga, pues se nos va cortando. El documento que se aprobó no era el documento que queríamos el movimiento vecinal en Málaga" (ex Concejal de IU y miembro de la Federación de AA.VV.). «Es importante que estén [otras asociaciones], pero que aquí si alguien había luchado por la participación eran las AA.VV., y por lo tanto entendíamos que eran las que tenían, un poco, que dirigir el proceso" (miembro de la Federación de AA.VV.).

${ }^{21}$ El mismo fenómeno parece producirse en lo que se refiere a las subvenciones. El gobierno municipal de Málaga no reduce en términos absolutos la cantidad de subvenciones que concede a las AA.VV., pero sí lo hace en términos relativos. Así, las Asociaciones de Vecinos pasan de recibir el 34 por 100 de las subvenciones en 1993 a recibir el 20,5 en 1995. Para el resto se producen ciertos incrementos (véase Navarro, 1998: 144). 


\section{FIGURA 3}

\section{Indice de Representación de las asociaciones en Plenos de Distrito en el municipio de Málaga (1996)}

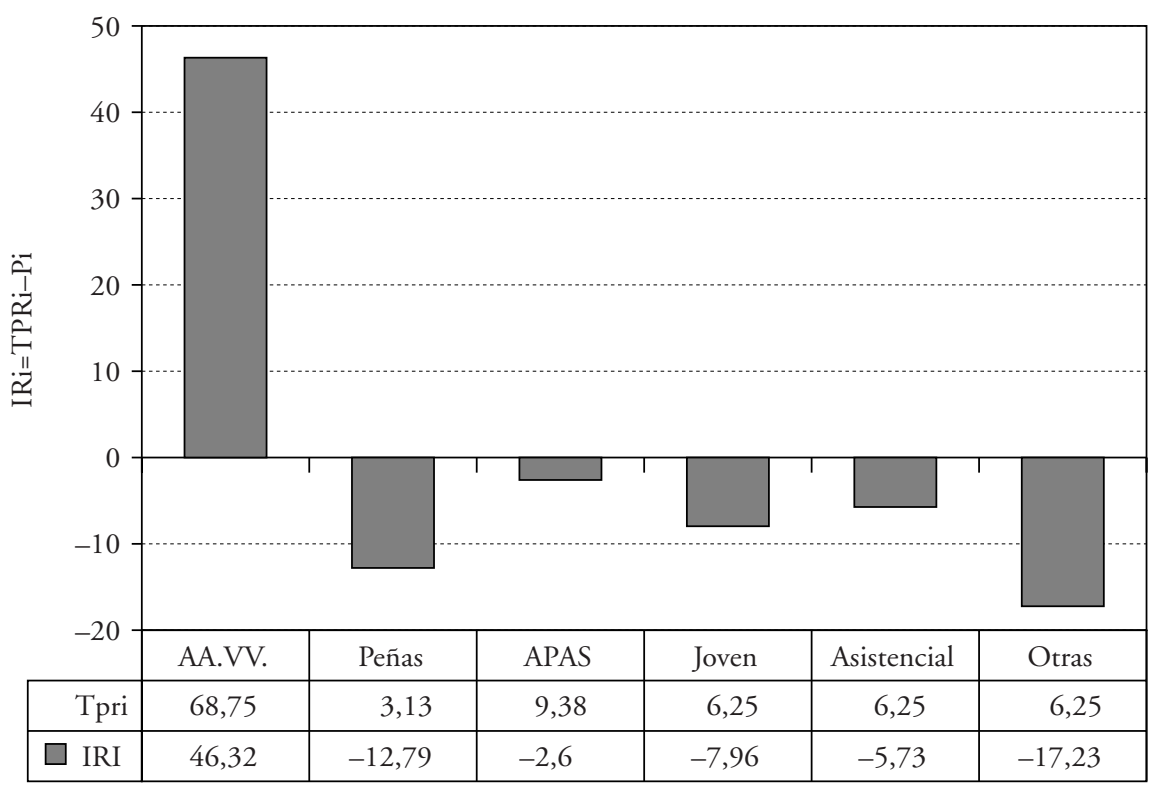

Tipo de colectivos

IRI: Índice de Representación del colectivo I en Pleno de Distrito. TPRI: Tasas de presencia del colectivo I en Pleno de Distrito.

FUENTE: Navarro (1998: 145).

Así pues, cabe destacar que en el caso de Málaga el gobierno actúa con la creencia de que las AA.VV. le son mayoritariamente adversas y que, por lo tanto, se halla en una situación subóptima para él. Por ello trata de pasar, sin conseguirlo, de una situación heredada en la que se fomentaba en especial a las AA.VV. (cuando PSOE y PCE gobernaban en coalición) a otra en la que estas últimas pierdan su representación mayoritaria en los Plenos de Distrito en favor de asociaciones que el ejecutivo cree más afines, como las APAS. 


\section{CONCLUSIÓN}

En el presente artículo hemos elaborado un modelo que trata de explicar el comportamiento de dos de los actores principales del sistema de interacción de una democracia local: el gobierno municipal y las asociaciones locales. Este modelo nos ha permitido dilucidar cómo se conducirá un gobierno municipal que actúa racionalmente si su interés consiste tanto en maximizar el voto como en abrir cauces a la participación ciudadana a través de asociaciones locales representativas de los intereses ciudadanos. Hemos intentado contrastar, además, el modelo formal con la realidad política de dos municipios concretos (Córdoba y Málaga) que durante una quincena pusieron en marcha políticas de democratización fomentando la participación en los asuntos municipales de asociaciones locales.

El fomento de la participación de los ciudadanos en la toma de decisiones públicas (la democratización de la democracia) a través de asociaciones que representen sus intereses, no sólo está determinado por el tamaño o la escala en que se desarrolla la democracia, sino también por la estructura del sistema político: en nuestro caso una democracia representativa de competencia entre partidos. Así, el hecho de que el principal interés de los partidos resida en maximizar el voto lleva aparejado que a menudo se tienda a crear un sistema clientelista de relación entre asociaciones locales y gobierno municipal. Escala y estructura del sistema serían, pues, las dos fuerzas que determinan la posibilidad real de establecer mecanismos institucionales que favorezcan una participación ciudadana plena y equitativa. De aquí cabe desprender la dificultad que entraña la puesta en marcha de experiencias de democracia participativa en el seno de la democracia representativa de competencia entre partidos. Dado ese marco institucional, el partido o la coalición en el gobierno municipal tienen un gran incentivo para actuar como si fueran compradores en un mercado de trastos. Para evitar ese comportamiento, los procesos de democratización de la democracia local deberían regularse de forma que estuviera asegurada la participación equitativa de todas las asociaciones en función de su representatividad. Sin embargo, es éste un problema de teoría normativa de la democracia que va más allá de los límites del presente trabajo. 


\section{BIBLIOGRAFÍA}

AKerlof, G. (1970): "The market for "lemons": quality uncertainty and the market mechanism», Quarterly Journal of Economics, 84, pp. 488-500.

Amodio, J. (1986): "The Spanish Communist Party and local government: An overall assessment», en B. Szajkowski (comp.), Marxist Local Governments in Western Europe and Japan, Londres: France Printer.

BLAIR, Ph. (1991): "Trends in local autonomy and democracy: reflections from an european perspective», en R. Batley y G. Stoker (eds.), Local Government in Europe. Trends and developments, Londres: MacMillam.

BolDUC, V. (1980): «Representation and legitimacy in neighborhood organizations: a case study", Journal of Voluntary Action Research, 9, pp. 165-178.

Boudon, R. (1981): La lógica de lo social, Madrid: Rialp.

Budge, I. (1996): The New Challenge of Direct Democracy, Londres: Polity Press.

Castells, M. (1986): La ciudad y las masas. Sociología de los movimientos sociales urbanos, Madrid: Alianza Editorial.

CENSIS (1991): "Dossier associazionismo: i nuovi canali di consenso e partecipazione», en Censis. Note e comenti, a. XXVIII, 3-4.

ClARK, T. N. (1995): «The local interests system: who governs and why?», University of Chicago, draft paper of FAUI project.

Clarke, S., y Gaile, G. (1989): «Moving towards entrepreneurial state and local economic development strategies: opportunities and barriers», Policy Studies Journal, 17, pp. 564-598.

Coleman, J. (1990): Foundations of Social Theory, Cambridge, Mass.: Harvard University Press.

Dahl, R., y Tufte, E. (1974): Size and Democracy, Oxford: Oxford Univesity Press.

DAsGupta, P. (1988): «Trust as a commodity», en D. Gambetta (comp.), Trust, Oxford: Blackwell.

Diani, M. (1992): «Social Movements as Networks», en M. Diani y R. Eyerman (eds.), Studiying Collective Action, Londres: Sage.

Downs, A. (1973): Teoría económica de la democracia, Madrid: Aguilar.

- (1991): "Una teoría económica de la acción política en una democracia», en J. Colomer (comp.), Lecturas de teoría politica positiva, Madrid: Instituto de Estudios Fiscales, pp. 263298.

Fundación EnCUENTro (1994): España 1994, Madrid: Fundación Encuentro.

- (1995): España 1995, Madrid: Fundación Encuentro.

GARDNER, R. (1996): Juegos para empresarios y economistas, Barcelona: Antoni Bosch Editor.

Gates, S., y Hume, B. (1997): Games, Information and Politics, Ann Arbor: The University of Michigan Press.

Gunther, R.; SANI, G., y SABAH, G. (1986): El sistema de partidos políticos en España: génesis y evolución, Madrid: CIS.

Hardin, R. (1982): Collective Action, Londres: The Johns Hopkins University Press.

Quantin, P. (1989): Local Politics and Participation in Britain and France, Cambridge: Cambridge University Press.

Linder, W. (1994): Swiss Democracy, Londres: St. Martin's Press.

Marwell, G., y Oliver, P. (1993): The Critical Mass in Collective Action, Cambridge: Cambridge University Press.

Navarro, C. (1998): El nuevo localismo, Córdoba: Diputación de Córdoba.

- (1999): El sesgo participativo, Madrid: CSIC.

OLIVER, P. (1984): "IIf you don't do it nobody else will": active and token contributors to local collective action", American Sociological Review, 49, pp. 601-610.

Parry, G.; Moyser, G., y Day, N. (1992): Political participation and Democracy in Britain, Cambridge: Cambridge University Press.

Parry, G., y Moran, M. (1994): Democracy and Democratization, Londres: Routledge.

RASMUSEN, E. (1989): Games and Information, Oxford: Blackwell. 
SARTORI, G. (1988): Teorías de la democracia, Madrid: Alianza Editorial.

SHUbiK, M. (1992): Economía política: un enfoque desde el punto de vista de la Teoría del Juego, México: FCE.

Thursz, D. (1972): "Community participation: should past be prologue?», American Behavioral Scientist, 15, pp. 733-748.

\begin{abstract}
We have often wished to see the municipal sphere as being, for reasons of scale, the most ideal for putting into practice citizen participation experiences that involve the democratization of representative democracy. From our point of view, scale is not, however, the only obstacle in the way of such democratizing processes. There are structural constrictions that are related to the nature of the democratic systems of authority existing between parties and which determine the preferences of the social protagonists in such a way that the democratization of local representative democracy through the channel of citizen participation becomes complex. This conclusion is reached after using the instruments of the games theory for an analysis of the system used for integration of protagonists of the model (municipal government and local associations). It seems that this system of interaction also possesses a series of characteristics that have a certain similarity to what is known in economics as a junk market. The abstract model is applied to the study of two specific cases: that of citizen participation experiences in the municipal districts of Cordoba and Malaga between 1979 and 1995 .
\end{abstract}


NOTAS DE INVESTIGACIÓN 